\title{
ANALYSIS OF IMAGE ENHANCEMENT METHODS FOR OUTDOOR-APPLICATIONS
}

\author{
Philipp Gujon, Wilfried Kubinger
}
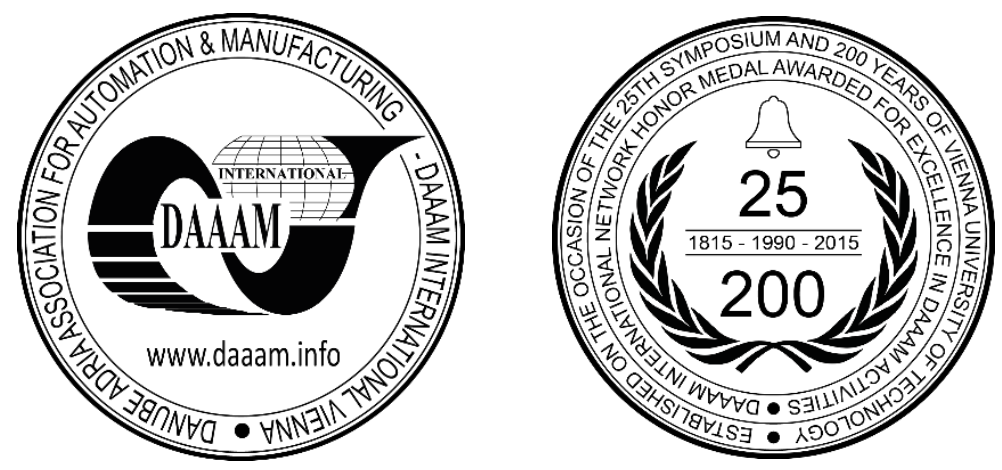

This Publication has to be referred as: Gujon, P[hilipp] \& Kubinger, W[ilfried] (2016). Analysis of Image Enhancement Methods for Outdoor-Applications, Proceedings of the 27th DAAAM International Symposium, pp.0040-0043, B. Katalinic (Ed.), Published by DAAAM International, ISBN 978-3-902734-08-2, ISSN 1726-9679, Vienna, Austria DOI: $10.2507 / 27$ th.daaam.proceedings.006

\begin{abstract}
For autonomous robots and driver assistance systems it is necessary to react adequately to sudden changes in the surrounding area. Camera-systems are often affected by environmental influences like sun light, shades or sunreflecting surfaces which makes it hard to obtain well exposed images for further utilization. In this paper, two algorithms for image enhancement are compared in several test scenes to examine their performance with regard to contrast enhancement and computing time. In comparison one off-the-shelf high dynamic range camera is tested, too. The results from this study show that histogram equalization works significantly faster than the successive mean quantization transform. Both algorithms are appropriate to be used for autonomous robots and driver assistance systems if the camera is incapable to high dynamic range images.
\end{abstract}

Keywords: High dynamic range imaging; successive mean quantization transform; histogram equalization; autonomous robots; driver assistance systems

\section{Introduction}

Regarding to the steady growth of autonomous machines in industry and agriculture it always becomes more inevitably to be able to react on many environmental influences. To solve this setting of tasks camera-systems are frequently used [1-2].

However, especially in outdoor applications it is hard for camera-systems to work adequately because of a huge variation in the objects appearance due to changing weather and light conditions. Performing the task automatically without human intervention is particularly hard in image processing. Many methods have been proposed to handle this challenge.

According to [3] these methods to achieve a contrast enhancement can be divided into two groups. One way to solve the problem is to take minimum 3 pictures of the same scene with different exposures and combine them to one well exposured picture with enhanced contrast. Different approaches and techniques have been suggested to solve this problem [4]-[6].

But in fast changing environments it is possible, that some fragments are not shown on the right place. This phenomenon is called "Ghost Effect". 
To prevent pictures with not trustworthy information a lot of algorithms for digital image processing and contrast enhancement have been proposed [7]-[11]. Especially automotive suppliers are very interested in video cameras which can cope with varying lightning conditions. For advanced driver assistance systems (ADAS) it is mandatory to be robust against direct sunlight or dark areas - e.g., when driving in or out of a tunnel [12]-[17].

\section{Contrast Enhancement}

Every digital image consists out of single pixels. In grey scale pictures each of these image points corresponds to a brightness value. Nowadays, there are different kind of sensors available. The difference between them is how many steps are between the brightest and the darkest point. An 8 bit sensor, for example, can distinguish the luminance of an image into 256 steps. According to these insights, a picture can be interpreted as a matrix of values in the area [0,255]. The graphical representation of the distribution of the image points with the prevailing intensities is called histogram.

This is the basis of the histogram equalization procedure. This method causes a contrast enhancement by an equipartition of the brightness-value distribution. The calculation occurs by means of accumulated sum of the pixels. This entails that those areas of the histogram with higher appearing grey values are stretched and those with smaller amount are compressed.

With this method it is possible to make the full use of the whole range of intensities in a picture. The result is a contrast enhancement. The histogram equalization automatically flattens and stretches the histogram and therefore, the dynamic range of the image.

However, the successive mean quantization transform perform an automatic structural breakdown of information and these characteristic make the transform interesting for automatic enhancement of any images [9]. The smallest unit of the arithmetic process is the Mean Quantization Unit (MQU). The SMQT function can be described by a binary tree of MQU's as it is shown in Figure 1.

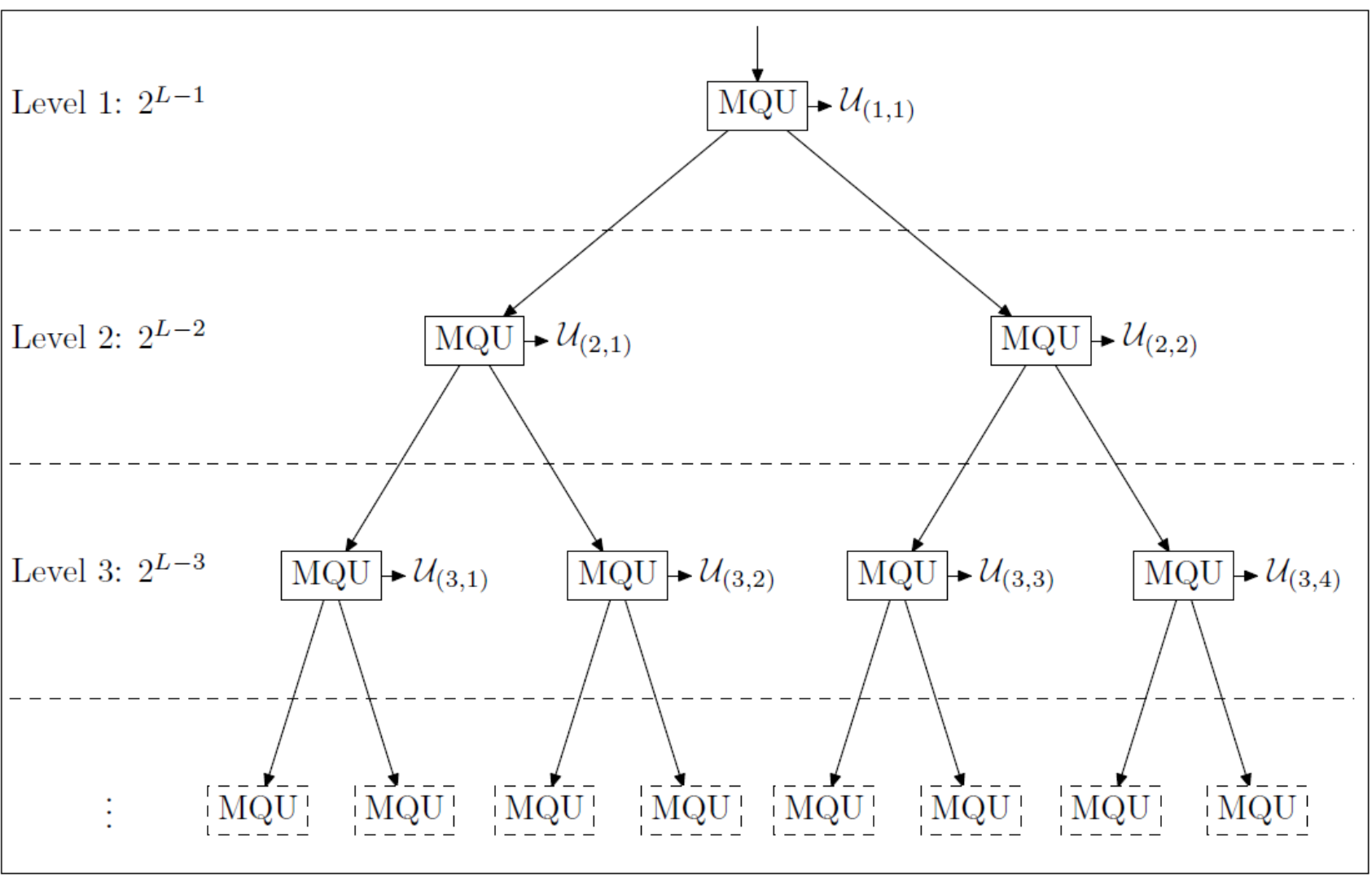

Fig. 1. The SMQT as a binary tree of MQU's (source: [7])

Histogram equalization, which was developed for image contrast enhancement, is similar to the successive mean quantization transform. However, both algorithms are used to enhance the luminance of low dynamic range images to extend the dynamic range.

\section{Experimental Results}

In fact that the histogram equalization and successive mean quantization transform algorithms calculate all pixels new in the picture, the computing time is given in $10^{-9} \mathrm{sec} / \mathrm{pixel}$ (ns/pixel). 
This allows a further objective comparison to different sized pictures. As time measurement the tic-toc function in MatLab on a computer with an i7-4700MQ 2,4GHz processor was used.

In Table 1 the arithmetic average of 20 procedures with 100 image calculations are listed. The computing time and the maximum possible picture rate with a picture format of $1024 \times 768$ pixels is shown, too.

\begin{tabular}{|c|c|c|}
\hline Method & $\begin{array}{c}\text { Computing time } \\
\text { [ns/pixel] }\end{array}$ & $\begin{array}{c}\text { Max. frame rate } \\
{[\mathbf{f p s}]}\end{array}$ \\
\hline SMQT grey level & 90,56 & 14 \\
\hline SMQT colour & 528,81 & 2 \\
\hline HE grey level & 2,2 & 574 \\
\hline HE colour & 50,66 & 25 \\
\hline
\end{tabular}

Table 1. Computing time and maximum frame rate of SMQT and HE.

The results of this comparison shows that the HE method is faster to compute than the SMQT method and therefore, it is possible to get higher frame rates which is necessary for fast changing environments.

Regarding to the fact that in grey-level pictures every pixel represents only the value of saturation, the computing time is much faster than for colour pictures. The tested algorithms were applied in images with different dynamism extent of the motive. The test scenarios contained under- and over- exposed pictures as well as backlight conditions to simulate any environmental factors like in real-world outdoor applications.

In Figure 2 the results of the image enhancement of a picture with poor contrast and for comparison an image of the same scene with a high dynamic range camera is shown. The differences are evident between images take with a customary industrial camera and a camera with raised dynamism extent as well as the contrast enhancement with the algorithms proposed on top.

Both methods for contrast enhancement deliver satisfactory results, nevertheless, the quality of the images is clearly worse than those of the HDR camera [18]. In closer consideration it is apparent that as a consequence of the contrast enhancement the white noise increases in the dark regions of the picture.

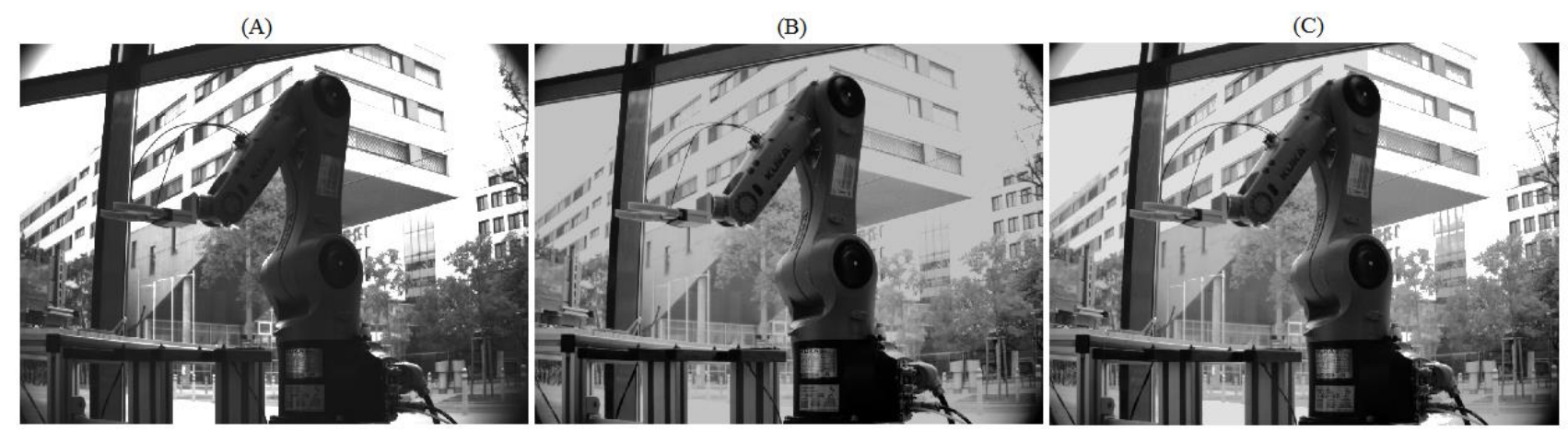

(D)

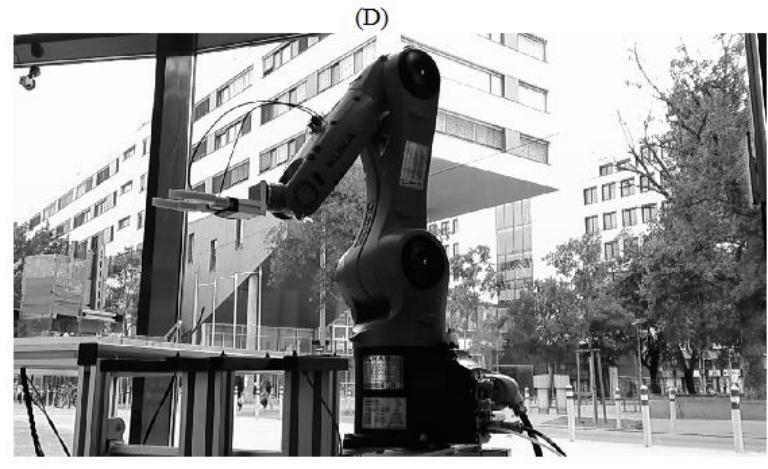

Fig. 2. (A) Low dynamic range image with back-light conditions. (B) The enhanced image with HE. (C) The enhanced image using the SMQT method. (D) High dynamic range image taken with an HDR-camera DFK AFU130-L53 from

The Imaging Source [18].

\section{Conclusion}

In this paper the histogram equalization and the successive mean quantization transform methods for contrast enhancement of a low dynamic range image were evaluated and a comparison with an HDR-camera was shown. Both algorithms for image enhancement were compared in several test scenes to examine their performance with regard to 
computing time and level of contrast enhancement. For comparison, the results of a high dynamic range camera were demonstrated, too.

We show, that the histogram equalization works significantly faster than the successive mean quantization transform.

Both algorithms deliver satisfying results in contrast enhancement but the SMQT shows more focus on details.

However, HDR-cameras provide excellent images even in dynamic environments.

\section{Acknowledgments}

This project has been funded in part by MA23 - City of Vienna within the Project Call 16-02 "Photonics: Foundations and industrial applications" and by the Austrian Security Research Programme KIRAS - an initiative of the Austrian Federal Ministry for Transport, Innovation and Technology (bmvit).

\section{References}

[1] Woeber, W.; Kefer, M.; Kubinger, W. \& Szuegyi, D. (2012). Evaluation of Daylight and Thermal Infra-Red based Detection for Platooning Vehicles, Annals of DAAAM for 2012 \& Proceedings of the 23rd International DAAAM Symposium, ISBN 978-3-901509-91-9, ISSN 2304-1382, pp 0719 - 0722, Editor B[ranko] Katalinic, Published by DAAAM International, Vienna, Austria.

[2] Spulak, D.; Otrebski, R \& Kubinger, W. (2015). Evaluation of PCA, LDA and Fisherfaces in Appearance-based Object Detection in Thermal Infra-red Images with Incomplete Data. Procedia Engineering, Volume 100, 2015 , pp. 1167-1173.

[3] Myszkowski, K.; Mantjuk, R. \& Krawczyk, G. (2008). High dynamic range video. Lectures on Computer Graphics and Animation, Morgan \& Claypool Publishers, 2008, 1, 1-158.

[4] Li, S.; Kang, X. \& Hu, J. (2013). Image fusion with guided filtering. Image Processing, IEEE Transactions on, 2013, 22, 2864-2875.

[5] Matsuoka, R.; Ishibashi, H.; Baba, T. \& Okuda, M. (2015). Multiple exposure integration for restoring all in-focus images. Image Processing (ICIP), 2015 IEEE International Conference, 2015, 4165-4169.

[6] Park, S. \& Kim, J. H. (2006). Wide dynamic range vision for robot eyes based on double capturing scheme sensors. IEEE Conference, 2006, 5, 1473-1476.

[7] Nilsson, M.; Dahl, M. \& Claesson, I. (2005). Gray-scale image enhancement using the SMQT. IEEE International Conference on Image Processing (ICIP).

[8] Im, J.; Jeon, J.; Hayes, M. \& Paik, J. (2011). Single image-based ghost-free high dynamic range imaging using local histogram stretching and spatially-adaptive denoising. Consumer Electronics, IEEE Transactions, 2011, 57, 14781484.

[9] Nilsson, M.; Dahl, M. \& Claesson, I. (2005). The Successive Mean Quantization Transform. IEEE Internation Conference on Acoustics, Speech and Signal Processing (ICASSP).

[10] Sahani, M.; Rout, S. K.; Mohan Satpathy, L. \& Patra, A. (2015). Design of an embedded system with modified contrast limited adaptive histogram equalization technique for real-time enhancement. International Conference on Communications and Signal Processing (ICCSP), 2015, 332-335.

[11] Li, C.-C.; Hsu, S.-W.; Shen, P.-C. \& Guo, J.-I. (2014). A single-camera high dynamic range technique by using contrast enhancement and exposure control. Asia-Pacific Signal and Information Processing Association (APSIPA).

[12] Prinet, V.; Wang, J., Lee, J., \& Wettergreen, D. (2016). 3D road curb extraction from image sequence for automobile parking assist system. In: Image Processing (ICIP), 2016 IEEE International Conference on. IEEE, pp. 3847-3851.

[13] Ziebinski, A.; Cupek, R., Erdogan, H., \& Waechter, S. (2016). A Survey of ADAS Technologies for the Future Perspective of Sensor Fusion. In: International Conference on Computational Collective Intelligence. Springer International Publishing, pp. 135-146.

[14] Song, Y. \& Liao, C. (2016). Analysis and review of state-of-the-art automatic parking assist system. In: Vehicular Electronics and Safety (ICVES), 2016 IEEE International Conference on. IEEE, pp. 1-6.

[15] Hertel, D. W. \& Chang, E. (2007). Image quality standards in automotive vision applications. In Proc. IEEE Intelligent Vehicles Symposium, pp. 404-409.

[18] Knoll, P. M. (2007). HDR vision for driver assistance. In High-Dynamic-Range (HDR) Vision, Springer Berlin Heidelberg, pp. 123-136.

[17] Hoefflinger, B. (2016). HDR-and 3D-Vision SensorsVision Sensors. In CHIPS 2020 Vol. 2, Springer International Publishing, pp. 201-209.

[18] http://www.theimagingsource.com, (2016). The Imaging Source, DFK AFU130-L53 Color Camera data sheet, Accessed on: 2016-09-26 